\title{
Stator Flux Based Model Reference Adaptive Observers for Rotor Position and Speed Estimation in Doubly-Fed Induction Machines
}

\author{
Ramu Nair. R, G. Narayanan \\ Department of Electrical Engineering, Indian Institute of Science, Bangalore, India - 560012
}

\begin{abstract}
Sensorless estimation of speed is preferred for Doubly-Fed Induction Generator (DFIG) used in wind energy conversion systems, in view of cost, maintenance, cabling and reliability issues. This paper proposes two stator flux based Model Reference Adaptive Observers (MRAO) for rotor position and speed estimation in doubly-fed induction machines. The cross product of reference and estimated stator flux linkage vectors is fed as controller input in the existing method, thereby making the observer model non-linear. The first proposed method, which is based on notch filtering, offers a performance comparable to that of the existing method, while reducing the number of voltage and current sensors required. The second method linearizes the observer model, leading to superior dynamic performance over the existing methods. This also simplifies the design of the controller. Simulations and experiments are performed on a 10 HP DFIG coupled to a Squirrel Cage Induction Motor (SCIM).

Index Terms-Doubly-fed induction generator, rotor position estimation, notch filter, controller design.
\end{abstract}

\section{INTRODUCTION}

Majority of the generators employed in wind energy industry are Doubly-Fed Induction Generators (DFIG) [1], [2]. The main advantage of DFIGs is the reduced power rating of the converters, resulting in cost reduction and improved efficiency [1]-[4]. The grid side converter performs DC link voltage control. The rotor side converter controls the active and reactive power injection in the grid connected mode, and the voltage and frequency control in the standalone mode. The rotor side current control loops require rotor position information for $d q$ to $A B C$ transformation. The rotor position information can be obtained through position sensors or estimated by sensorless algorithms [5]-[11]. There are several advantages for sensorless rotor position estimation such as lower cost, lesser maintenance, and higher reliability [5]. Most sensorless methods are based on either Model Reference Adaptive Observers (MRAO) [5]-[9] or Phase Locked Loops (PLL) [10] or high frequency signal injection [11], [12]. A speed sensorless method for control of DFIG without flux estimation can be found in [13]. MRAOs are easier to implement and estimate speed accurately even during speed transitions between subsynchronous and supersynchronous speeds [9]. MRAO could

Financial support to this research was provided by Central Power Research Institute (CPRI), Ministry of Power, Government of India, under a project titled Power conversion, control and protection technologies for microgrid be based on stator flux, rotor current, torque or reactive power [5]. This paper proposes two stator flux based MRAOs, having significant advantages over the existing methods. The first proposed method achieves similar performance to the existing method, while requiring fewer number of sensors. The second proposed method aligns the reference and estimated stator flux linkage vectors by feeding the linearized error input to the controller, following similar approach as in [7], where the reference and the estimated rotor current vectors are aligned through controller action. Linearization results in superior dynamic performance and straight-forward controller design.

To begin with, existing stator flux based MRAO is reviewed in section II. Section III describes notch filter based MRAO, which achieves similar performance as the existing observer with reduced number of sensors. The design and implementation of notch filter, followed by small signal analysis and controller design for the proposed scheme, are also included in this section. Linearized observer is explained in section IV which has superior dynamic performance compared to existing methods. Linearization also facilitates a straight-forward controller design. The dynamic performance of the existing and the proposed observers are compared first using simulations, which are given in section V. Experimental results for dynamic performance of the different observers are included in section VI. This is followed by conclusion in section VII.

\section{Review Of Existing Stator Flux Based MRAO}

A block diagram of the existing stator flux based MRAO (SF-MRAO) [5] is shown in Fig. 1. The reference stator flux linkage vector, $\lambda_{s, r e f}^{s}$, is obtained from the stator side voltage equation. The estimated vector, $\lambda_{s, e s t}^{s}$, is generated from the sensed stator and rotor currents, following (1). The transformation of rotor current vector, $i_{r}^{r}$, to stator reference frame, $\boldsymbol{i}_{r}^{s}$, requires information of rotor position, $\theta_{A a}$. The controller input is chosen to be the cross product of reference and estimated stator flux linkage vectors. The controller output is the rotor speed, $\omega_{A a}$. The integration of the rotor speed yields rotor position, $\theta_{A a}$. This is fed to the transformation block as indicated in Fig. 1. At steady state, the reference and estimated vectors are aligned resulting in the estimated rotor speed and position. 


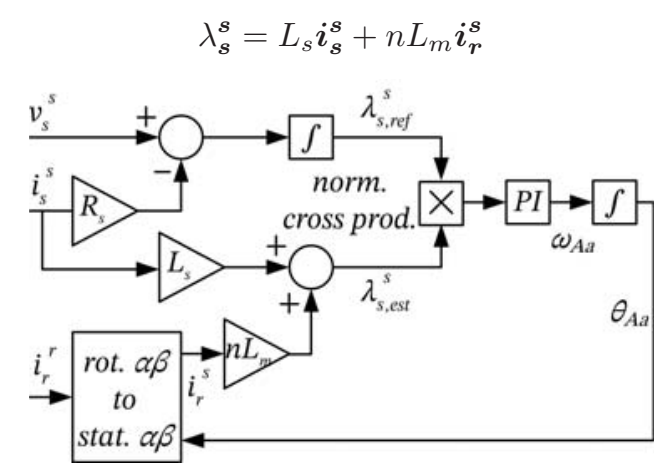

Fig. 1: Existing stator flux based MRAO

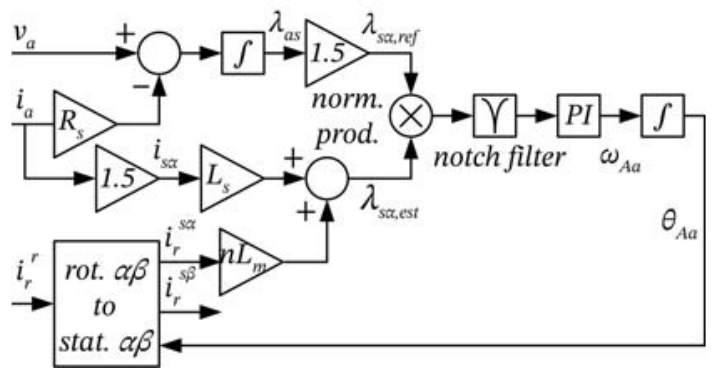

Fig. 2: Proposed notch filter based MRAO

\section{Proposed Method 1: Notch Filter Based MRAO}

The proposed notch filter based MRAO is shown in Fig. 2. This method aligns the reference and estimated stator flux linkage vectors through their respective $\alpha$ - axis components. The advantage of using the $\alpha$ - axis components is in having reduced number of voltage and current sensors. The $\alpha$ component of a three phase quantity is proportional to the corresponding $a$ - phase quantity in balanced condition. Hence, for generating the $\alpha$ - axis component of the reference stator flux linkage vector, $\lambda_{s \alpha, r e f}$, only $v_{a}$ and $i_{a}$ need to be sensed, unlike in the existing method, where two phase voltages and two phase currents have to be sensed for generating the reference vector, $\lambda_{s, r e f}^{s}$. The instantaneous product of $\lambda_{s \alpha, r e f}$ and $\lambda_{s \alpha, e s t}$ is taken, resulting in a signal with sum and difference of the frequencies of the $\alpha$ - components. Since the stator side of the DFIG is maintained at $50 \mathrm{~Hz}$, the frequency of $\lambda_{\text {sa,ref }}$ is also at $50 \mathrm{~Hz}$. Hence, the sum of the two frequencies is $100 \mathrm{~Hz}$ at steady state. This is filtered using a notch filter at $100 \mathrm{~Hz}$. Note that a low pass filter at $10 \mathrm{~Hz}$ was not found to be feasible. The design and implementation of the notch filter are explained in the following subsection. The residual signal with the difference of the two frequencies is chosen as the controller input. The controller tries to attenuate this error input to zero, thereby aligning the two $\alpha$ - axis components. The PI output, $\omega_{A a}$, is integrated to obtain $\theta_{A a}$ which is fed to the transformation block as in the existing method.

\section{A. Design of Notch Filter}

The notch filter transfer function, $N(s)$, is implemented based on high gain feedback at the desired frequency $\left(\omega_{n}\right)$ to

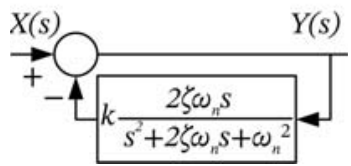

(a) Notch filter, N(s)

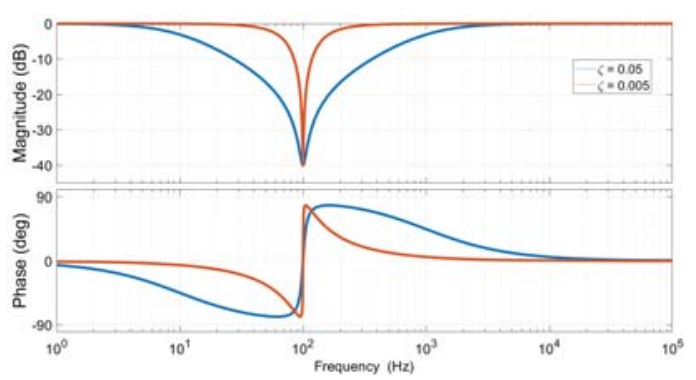

(b) Bode plot of notch filter for $\zeta=0.005$ and $\zeta=0.05$, at $k=100$

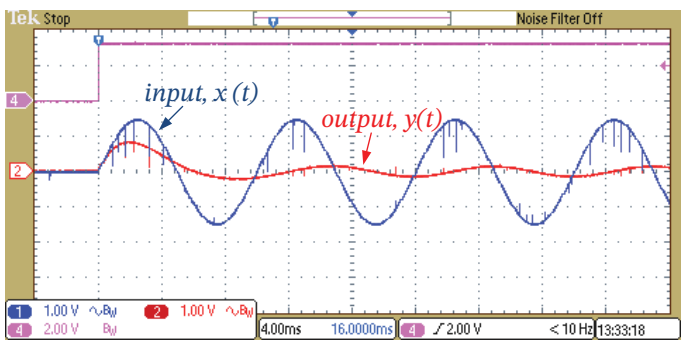

(c) Performance of notch filter for $\zeta=0.005, k=100$

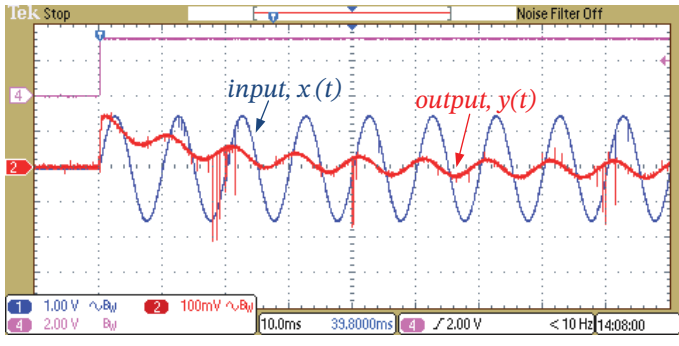

(d) Performance of notch filter for $\zeta=0.05, k=100$

Fig. 3: Notch filter design and performance

be attenuated (Fig. 3(a)). The bode plots of $N(s)$ for $\zeta=0.005$ and $\zeta=0.05$ at gain, $k=100$ are given in Fig. 3(b). Since there is drift in resonant frequency upon discretisation, lower values of damping factor (i.e. $\zeta \approx 0$ ) lead to poor attenuation. The attenuation achieved for $\zeta=0.005$ and $\zeta=0.05$ at gain $k=100$ are $7 \%$ and $70 \%$, respectively (Figs. 3(c), 3(d)).

\section{B. Small signal analysis and controller design}

Let the normalised instantaneous product of the two ac waveforms, $\lambda_{s \alpha, r e f}$ and $\lambda_{s \alpha, e s t}$, at the output of the multiplier be,

$$
\begin{aligned}
p & =\sin \left(\theta_{x}\right) \sin \left(\theta_{y}\right) \\
& =\frac{\cos \left(\theta_{x}-\theta_{y}\right)+\cos \left(\theta_{x}+\theta_{y}\right)}{2}
\end{aligned}
$$




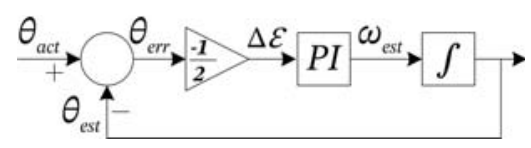

Fig. 4: Block diagram for controller design of proposed notch filter based SF-MRAO

where $\theta_{x}$ and $\theta_{y}$ are the phases of $\lambda_{s \alpha, \text { ref }}$ and $\lambda_{s \alpha, e s t}$ respectively. After filtering of $\cos \left(\theta_{x}+\theta_{y}\right)$ component, the output of the notch filter at steady state is,

$$
\varepsilon=\frac{\cos \left(\theta_{\text {err }}\right)}{2}, \quad \text { where } \theta_{\text {err }}=\theta_{x}-\theta_{y}
$$

By considering $\triangle \theta_{\text {err }}$ and $\triangle \varepsilon$ about $\theta_{\text {err }, s s}$ and $\varepsilon_{s s}$, respectively,

$$
\begin{aligned}
\varepsilon_{s s}+\triangle \varepsilon & =\frac{\cos \left(\theta_{e r r, s s}+\triangle \theta_{e r r}\right)}{2} \\
& =\frac{\cos \left(\theta_{e r r, s s}\right) \cos (\triangle \varepsilon)-\sin \left(\theta_{e r r, s s}\right) \sin \left(\triangle \theta_{e r r}\right)}{2}
\end{aligned}
$$

The steady state error input to PI controller, $\varepsilon_{s s}$, is zero,

$$
\therefore \varepsilon_{s s}=0, \quad \theta_{\text {err }, s s}=\frac{\pi}{2}
$$

Substituting these values in the previous equation and simplifying,

$$
\begin{aligned}
\Delta \varepsilon & =\frac{-\sin \left(\Delta \theta_{e r r}\right)}{2} \\
& \approx \frac{-\Delta \theta_{e r r}}{2} \quad \because \Delta \theta_{e r r} \approx 0
\end{aligned}
$$

The open loop small signal transfer function (Fig. 4) is given by,

$$
G(s)=-\frac{1}{2} \frac{s k_{p}+k_{i}}{s^{2}}
$$

Substituting $s=j \omega$,

$$
G(j \omega)=\frac{1}{2} \frac{k_{i}+j \omega k_{p}}{\omega^{2}}
$$

Since the magnitude of $G(s)$ at the desired gain crossover frequency, $\omega_{g c}$, is unity, the following equation can be formed.

$$
\left|G\left(j \omega_{g c}\right)\right|=\frac{1}{2} \frac{\sqrt{k_{i}^{2}+\left(\omega_{g c} k_{p}\right)^{2}}}{\omega_{g c}^{2}}=1
$$

In-order to meet the phase margin (PM) requirement, the phase of $G(s)$ at $\omega_{g c}$ should be $-\pi+P M$. Hence,

$$
\measuredangle G\left(j \omega_{g c}\right)=\tan ^{-1}\left(\frac{\omega_{g c} k_{p}}{k_{i}}\right)=-\pi+P M
$$

Solving (4) and (5) for $k_{i}$ and $k_{p}$ yields,

$$
\begin{gathered}
k_{i}=\frac{2 \omega_{g c}^{2}}{\sqrt{1+(\tan P M)^{2}}} \\
k_{p}=k_{i} \frac{\tan P M}{\omega_{g c}}
\end{gathered}
$$

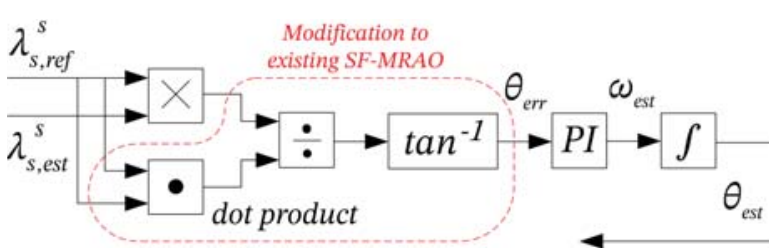

(a)

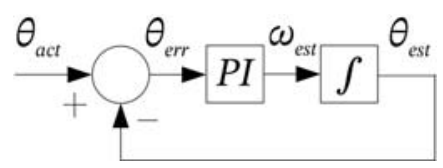

(b)

Fig. 5: Proposed linearized MRAO (a) linearization of existing SF-MRAO, (b) Block diagram for design of controller of proposed linearized observer

\section{Proposed Method 2: Linearized MRAO}

The existing methods align the reference and estimated vectors by feeding their normalized cross product $\left(\sin \theta_{\text {err }}\right)$ to the PI input, where $\theta_{\text {err }}$ is the angle between the two vectors. Since the observer model is non-linear, small signal analysis is necessary for controller design. The observer model can be linearized by feeding $\theta_{\text {err }}$ to the PI input instead of $\sin \theta_{\text {err }}$. Division of cross product by dot product yields $\tan \theta_{\text {err }}$, from which, $\theta_{\text {err }}$ can be obtained by taking its inverse tangent [7]. The modification done to the existing model to obtain linearized MRAO is highlighted in Fig. 5(a). Linearization also makes controller design straight-forward (Fig. 5(b)), without any small signal analysis, as in the existing methods.

\section{Simulation Results}

The dynamic performance of the existing and proposed SF-MRAOs are compared through simulations. The controller parameters for the existing and proposed linearized SF-MRAO are as suggested in [5] and [7], respectively. The controller design for the proposed notch filter based SF-MRAO is given in section III (B). The bandwidth and phase margin specifications for controller design are $10 \mathrm{~Hz}$ and $60^{\circ}$, respectively, for all observers. Simulation results illustrating the dynamic performance of the existing and proposed observers are shown in Fig. 6.

The rotor of the DFIG is assumed to be driven at a constant speed of $20 \mathrm{~Hz}$. The PI controller is enabled at the same error input (0.7) for all observers. The comparison of dynamics in the estimation of rotor speed and position are shown in Fig. 6(a) and Fig. 6(d), respectively. The error input to the controller tends to zero following different dynamics for the existing and proposed observers, and these are indicated in Fig. 6(b). The dynamics with which the $\alpha$-component of estimated stator flux linkage vector aligns with that of the reference stator flux linkage vector is given in Fig. 6(c). It can be observed from the simulation results that the existing observer and notch filter based observer have similar dynamic performances. The estimated rotor speeds of both these observers have similar 



(a) Comparison of dynamics in estimation of speed


(b) Comparison of dynamics of error input to the PI controller tending to zero


(c) Comparison of dynamics of $\alpha$-component of reference and estimated stator flux linkage vectors
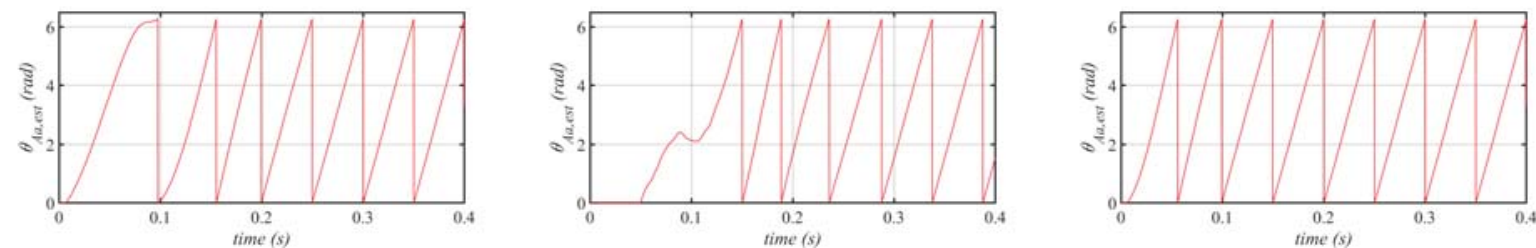

(d) Comparison of dynamics in estimation of rotor position

Fig. 6: Simulation results of dynamic performance for the same error input, $\varepsilon$, at the initiation of control, of existing SF-MRAO, proposed notch filter based SF-MRAO, proposed linearized SF-MRAO, respectively

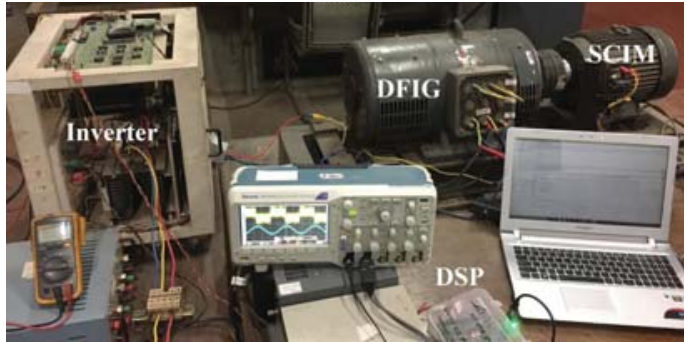

Fig. 7: Experimental setup

oscillations until their controller inputs are almost driven to zero at steady state, in $0.25 \mathrm{~s}$. For the proposed linearized observer, the error input tends to zero in $0.15 \mathrm{~s}$. Also, the estimated rotor speed has reduced oscillations in the proposed linearized observer. These simulation results match closely with the experimental results given in the next section.

\section{EXPERIMENTAL RESULTS}

Experiments are conducted on a 10 HP DFIG coupled to a Squirrel Cage Induction Machine (SCIM) at no load. The parameters of the 10 HP DFIG are tabulated in Table 1. The
TABLE I: Parameters of 10 HP DFIG

\begin{tabular}{|c|c|}
\hline Parameter & Value \\
\hline$R_{s}$ & $0.77 \Omega$ \\
\hline$R_{r}$ & $0.16 \Omega$ \\
\hline$L_{m}$ & $230 \mathrm{mH}$ \\
\hline$L_{l s}$ & $4.5 \mathrm{mH}$ \\
\hline$L_{l r}$ & $1.1 \mathrm{mH}$ \\
\hline turns ratio, $n$ & 3 \\
\hline
\end{tabular}

motor is run at $20 \mathrm{~Hz}$ through $\mathrm{V} / \mathrm{F}$ control. In-order to maintain stator side of DFIG at $50 \mathrm{~Hz}$, the excitation on the rotor side is $30 \mathrm{~Hz}$. MRAO bandwidth is at $10 \mathrm{~Hz}$ for all methods. MRAOs are implemented on TMS320F28377S DSP. A part of the hardware setup is shown in Fig. 7. Experimental comparison of dynamic performance of the different observers is indicated in Figs. 8 and 9. It can be observed from these figures that the existing observer and notch filter based observer have similar dynamic performances. Both these observers exhibit oscillations in the estimated rotor speed before settling in $0.22 \mathrm{~s}$. The dynamic performance of the linearized observer is superior to the existing and notch filter based observers with reduced oscillations and faster settling time of $0.12 \mathrm{~s}$. 


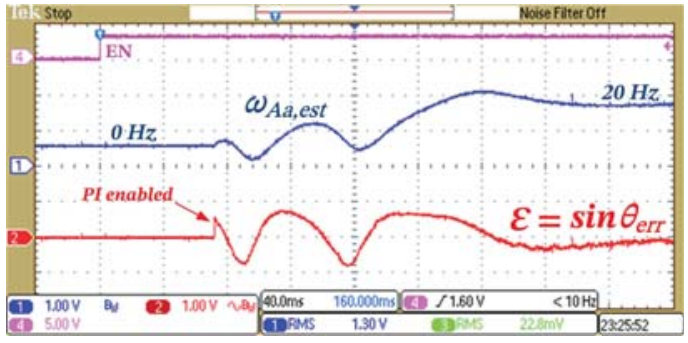

(a)

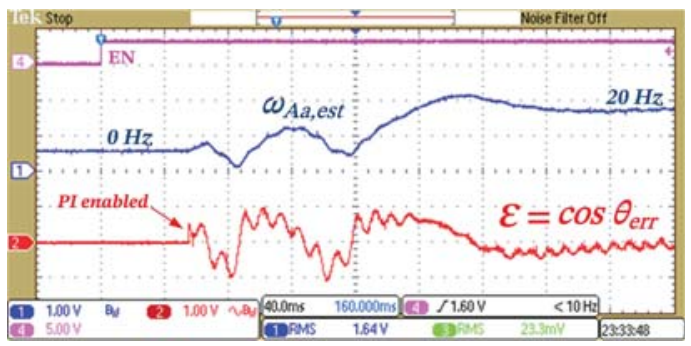

(b)

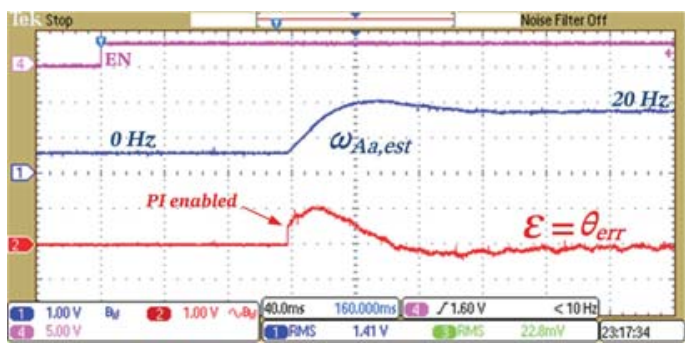

(c)

Fig. 8: Experimental comparison of dynamic performance for the same error input, $\varepsilon$, at the initiation of control: (a) existing SF-MRAO , (b) proposed notch filter based MRAO, (c) proposed linearized observer; trace (1) - dynamics of speed estimation, trace (2) - error input to controller

\section{CONCLUSION}

This paper proposes two stator flux based MRAOs for rotor position and speed estimation in DFIG. Experimental results show similar performances of the existing observer and proposed notch filter based observer, with the latter requiring fewer number of voltage and current sensors. The design and performance of the notch filter are discussed. The small signal analysis and controller design for the proposed notch filter based observer are explained. The dynamic performance of the linearized observer is superior to those of the existing and proposed notch filter based observers in terms of settling time and rotor speed oscillations, as validated by simulations and experiments. Linearization also simplifies controller design by eliminating the need for small signal analysis.

\section{REFERENCES}

[1] F. Blaabjerg and K. Ma, "Wind energy systems," Proceedings of the IEEE, vol. 105, no. 11, pp. 2116-2131, Nov. 2017.

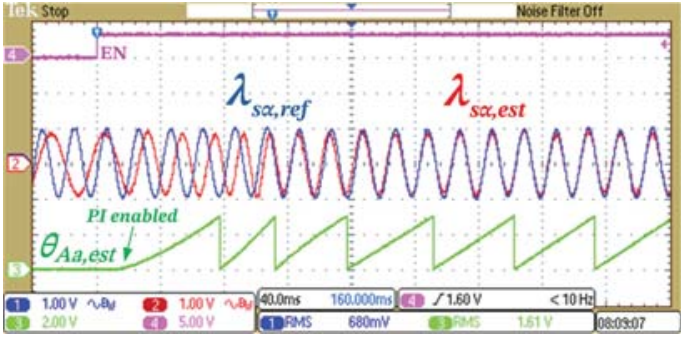

(a)

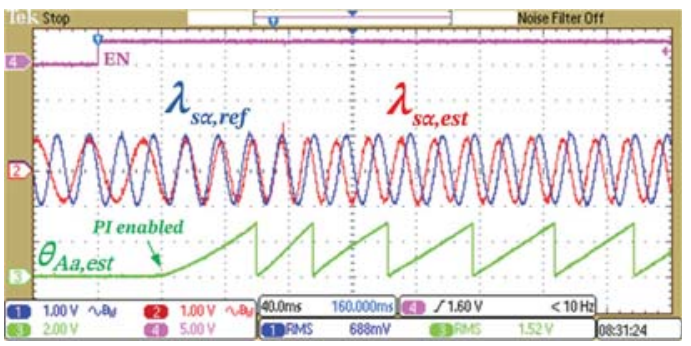

(b)



(c)

Fig. 9: Experimental comparison of dynamic performance for the same error input, $\varepsilon$, at the initiation of control: (a) existing SF-MRAO, (b) proposed notch filter based MRAO, (c) proposed linearized observer; traces $(1,2)$ - dynamics of alignment of $\alpha$-components of reference and estimated stator flux linkage vectors, trace (3) - estimated rotor position

[2] G. Abad, J. Lopez, M. Rodriguez, L. Marroyo, and G. Iwanski, DoublyFed Induction Machine: Modeling and Control for Wind Energy Generation. Hoboken, NJ, USA: Wiley, Oct. 2011.

[3] H. Polinder, J. A. Ferreira, B. B. Jensen, A. B. Abrahamsen, K. Atallah and R. A. McMahon, "Trends in wind turbine generator systems," IEEE Journal of Emerging and Selected Topics in Power Electronics, vol. 1, no. 3, pp. 174-185, Sept. 2013.

[4] F. Blaabjerg and K. Ma, "Future on power electronics for wind turbine systems," IEEE Journal of Emerging and Selected Topics in Power Electronics, vol. 1, no. 3, pp. 139-152, Sept. 2013.

[5] R. Cardenas, R. Pena, J. Clare, G. Asher and J. Proboste, "MRAS observers for sensorless control of doubly-fed induction generators," IEEE Transactions on Power Electronics, vol. 23, no. 3, pp. 1075-1084, May 2008.

[6] L. Lu, N. F. Avila, C. Chu and T. Yeh, "Model reference adaptive backelectromotive-force estimators for sensorless control of grid-connected DFIGs," IEEE Transactions on Industry Applications, vol. 54, no. 2, pp. 1701-1711, March-April 2018.

[7] R. R. Nair and G. Narayanan, "Modified model reference adaptive observer for rotor speed and position estimation in wound rotor induction machine," IEEE PES Asia-Pacific Power and Energy Engineering Conference (APPEEC), Bangalore, 2017, pp. 1-6.

[8] C. Lascu, I. Boldea, F. Blaabjerg and W. Chen, "A class of flux observers 
for doubly-fed induction generators used in small power wind generation systems," IEEE Energy Conversion Congress and Exposition, Denver, CO, 2013, pp. 2289-2295.

[9] M. Pattnaik and D. Kastha, "Adaptive speed observer for a stand-alone doubly-fed induction generator feeding nonlinear and unbalanced loads," IEEE Transactions on Energy Conversion, vol. 27, no. 4, pp. 1018-1026, Dec. 2012

[10] B. Mwinyiwiwa, Y. Zhang, B. Shen and B. Ooi, "Rotor position phase-locked loop for decoupled PQ control of DFIG for wind power generation," IEEE Transactions on Energy Conversion, vol. 24, no. 3, pp. 758-765, Sept. 2009

[11] D. D. Reigosa, F. Briz, C. Blanco Charro, A. Di Gioia, P. Garca and J. M. Guerrero, "Sensorless control of doubly-fed induction generators based on rotor high-frequency signal injection," IEEE Transactions on Industry Applications, vol. 49, no. 6, pp. 2593-2601, Nov.-Dec. 2013.

[12] D. D. Reigosa, F. Briz, C. Blanco and J. M. Guerrero, "Sensorless control of doubly-fed induction generators based on stator high-frequency signal injection," IEEE Transactions on Industry Applications, vol. 50, no. 5, pp. 3382-3391, Sept.-Oct. 2014.

[13] G. D. Marques and D. M. Sousa, "Air-gap-power-vector-based sensorless method for DFIG control without flux estimator," IEEE Transactions on Industrial Electronics, vol. 58, no. 10, pp. 4717-4726, Oct. 2011. 